[Aus dem Kaiserl. Institut für Infektionskrankheiten zu Tokio.] (Direktor: Prof. Dr. S. Kitasato.)

\title{
Bakteriologische Untersuchungen der Hausgenossen von Diphtheriekranken. Von
}

Dr. C. Nishino, Asaistenten am Institut.

Es ist eine wohlbekannte Tatsache, daB Bazillenträger bei einer Epidemie der betreffenden Krankheit eine große Rolle spielen. Besonders haben solche Infektionskrankheiten eine sehr groBe Bedeutung, bei welchen die Ansteckung nicht durch Vermittelung von leblosen Gegenständen, sondern hauptsächlich direkt durch Menschen gesebieht. Die Diphtherieinfektion ist hierfür ein gutes Beispiel.

Flūgge (1) hatte schon betont, einerseits auf dem biologischen Verhalten des Diphtheriebacillus und andererseits auf seiner Erfahrung über die Diphtherieepidemie in Breslau (1886 bis 1890) fußend, daB die Übertragung des Diphtheriebacillus in den meisten Fällen nicht durch leblose Gegenstände, sondern direkt durch Menschen geschieht. Scheller (2) sagt in seiner neuesten Monographie ebenfalls: „Die Ubertragung der Diphtherie geschieht fast ausschlieBlich direkt von Mensch zu Mensch."

Es gibt zahlreiche Mitteilungen [Neisser (3), Fibiger (4), Aaser (5), Cuno (6), Fisher (7) usw.] über Diphtheriebazillenträger, die, wegen ihres Gesundbleibens unbeachtet geblieben, eine anscheinend autochthone Epidemie verursachten. Ich selbst konnte auch im vorvorigen Jahre einen derartigen Fall beobachten, bei welchem eine Kinderwärterin einer adeligen Familie die Hausfrau mit drei Kindern, also im ganzen vier Personen, 
hintereinander innerhalb eines Jahres infizierte trotz einer jedesmal streng durchgeführten Desinfektion des Zimmers und Verbrennens der Kleidung der Kranken, bis weitere Infektionen erst durch die Absonderung jener Wärterin sistiert wurden.

Gerade damals hatte Hr. Direktor Prof. Kitasato mich angeregt, eine systematische bakteriologische Massenuntersuchung bei sämtlichen gesunden Hausgenossen von Diphtheriekranken zu unternehmen; hier möchte ich mir erlauben, ihm für die gütige Überlassung des Materials und seine freundliche Leitung meinen herzlichen Dank auszusprechen.

\section{Untersuchungsmethode.}

Sämtliche Familienglieder der Diphtherielranken, welche im Spital unseres Instituts aufgenommen worden waren, wurden als verdächtige Bakterienträger untersucht. War die Diagnose auf Diphtherie oder Krupp durch bakteriologische Untersuchung der Pseudomembran bzw. des Rachenschleims sichergestellt, so setzte ich mich mit der Familie in Verbindung und machte dort bei sämtlichen Familiengliedern Probeentnahme; war diese aus irgend einem Grunde durch mich nicht angängig, so wurde sie von seiten der Herren Kollegen in liebenswürdiger Weise ausgeführt, wofür ich ihnen hier meinen besten Dank ausspreche.

Die bakteriologische Untersuchung der entnommenen Proben erfolgte so, daB eine Loefflersehe Serumplatte mit dem mittels eines Wattetampons (an einem Glasstäbchen befestigt und vorher aseptisch gemacht) entnommenen Rachen- und Mandelschleim bestrichen wurde. Sodann wurde einen Tag später zunächst die mikroskopische Untersuchung der Kulturen vorgenommen, und aus denjenigen Bakterienkolonien, welche wenigstens mikroskopisch als Diphtherie anzusprechen waren, gleich eine Reinkultur mittels schiefer Serumröhrchen hergestellt. Die sichere Diagnose der Diphtheriebazillen habe ich erst dann gestellt, wenn eine Ose dieser Reinkultur Meerschweinchen bei der subkutanen Injektion unter den typischen pathologisch-anatomischen Veränderungen innerhalb einiger Tage getötet hatte. Bei den durch diese Methode festgestellten Bazillenträgern wurde, soweit die Cmstände es erlaubten, in Intervallen von je zwei bis vier Tagen dieselbe bakteriologische Untersuchung wiederholt, bis sie endlich zweimal hintereinander negativ ausfiel.

\section{Resultat.}

Meine Untersuchung über die Bazillenträger erstreckt sich vom Sep. tember vorvorigen Jahres ununterbrochen bis zum Februar dieses Jahres, also etwa anderthalb Jahre hindurch. In diesem Zeitraum konnte ich im 
ganzen 127 Familien von Diphtheriekranken mit 665 Individuen untersuchen, die Kranken selbst nicht eingerechnet. Unter diesen 665 befanden sich 41, also 6.2 Prozent sämtlicher Familienmitglieder, bei denen sich Diphtheriebazillen im Schlunde nachweisen ließen.

Wenn ich jetzt einige Literaturangaben über Massenuntersuchungen auf Diphtheriebazillenträger anführen darf, so fand Loeffler (8) unter 160 Schulkindern 2 (1.3 Prozent), Müller (9) unter 100 Kindern in der Kinderabteilung der Heubnerschen Klinik 24 (24 Prozent), Ustredt (10) unter 4277 Schulkindern 191 (4.5 Prozent), Fisher (7) unter 4081 Psychosen im Connecticut Hospital 92 (2 Prozent), Aaser (5) unter 89 Soldaten in einer Kaserne in Christiania 17 (19 Prozent) und endlich Fibiger (4) unter 134 Schülern in einem Gymnasium 10 (unter 8 Prozent) solcher Träger.

Die Untersuchungen dieser Autoren sind, wie erwähnt, ausschlieBlich auf eine besondere Ortlichkeit, wie Schule, Kaserne oder Hospital besehränkt, während nur Kober (11) die Untersuchung systematisch bei sämtlichen Familiengliedern Diphtheriekranker unternommen und unter 123 Individuen 10 (8 Prozent) gefunden hatte. Seine Untersuchung ist daher mit der meinigen gut vergleichbar.

Zur leichteren Übersicht habe ich die näheren Ergebnisse über meine 41 Fälle in nachstehender Tabelle kurz zusammengefabt.

Tabelle.

\begin{tabular}{|c|c|c|c|c|c|c|}
\hline Nr. & $\begin{array}{c}\text { Name des } \\
\text { Untersuchten } \text {. } \\
\text { seine Familien- } \\
\text { beziehung zum } \\
\text { Kranken } \\
\end{array}$ & 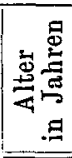 & $\begin{array}{l}\text { Wichtige } \\
\text { Anamnese }\end{array}$ & $\begin{array}{l}\text { Lokaler } \\
\text { Befund des } \\
\text { Rachens }\end{array}$ & Behandlnng & $\begin{array}{l}\text { Daner der } \\
\text { Anwesen- } \\
\text { heit des } \\
\text { Bacillus } \\
\text { in Tagen }\end{array}$ \\
\hline 1 & $\begin{array}{l}\text { Katsu, } \\
\text { Dienerin }\end{array}$ & 44 & $\begin{array}{l}\text { eigenes Kind } \\
\text { littvor4Jahren } \\
\text { an Diphtherie }\end{array}$ & normal & $\begin{array}{c}\text { Inj.v. I500 E.D.A. } \\
\text { Bepins. mit } \\
\text { Arg. nitr. }\end{array}$ & 12 \\
\hline 2 & $\begin{array}{c}\text { Suye, } \\
\text { Schwester }\end{array}$ & 8 & $\begin{array}{l}\text { vor } 3 \text { Jahren } \\
\text { Diphth.-Erkr. } \\
\text { durchgemacht }\end{array}$ & $"$ & desgl. & 11 \\
\hline 3 & $\begin{array}{c}\text { Taki, } \\
\text { Dienerin }\end{array}$ & 22 & $\begin{array}{c}\text { litt niemals } \\
\text { an Rachen- } \\
\text { krankheit }\end{array}$ & $\begin{array}{l}\text { nur leicht } \\
\text { injiziert }\end{array}$ & ," & 9 \\
\hline 4 & $\begin{array}{c}\text { Su, } \\
\text { Mutter }\end{array}$ & 37 & desgl. & $\begin{array}{l}\text { Mandeln } \\
\text { leicht inj. } \\
\text { u. angeschw. }\end{array}$ & nicht behandelt & 12 \\
\hline 5 & $\begin{array}{c}\text { Yuki, } \\
\text { Selnwester }\end{array}$ & 6 & " & normal & $\begin{array}{l}\text { Inj. } \\
1500 \text { E. D. A. }\end{array}$ & 14 \\
\hline 5 & $\begin{array}{l}\text { Shiro, } \\
\text { Bruder }\end{array}$ & 7 & " & $\begin{array}{c}\text { nux leicht } \\
\text { injiziert }\end{array}$ & $\begin{array}{l}\text { Inj.v. } 1500 \text { E.D.A. } \\
\text { Bepins, mit } \\
\text { Arg. nitr. }\end{array}$ & 6 \\
\hline 7 & $\begin{array}{c}\text { Ine, } \\
\text { Schwester }\end{array}$ & 3 & $\begin{array}{c}\text { nichts } \\
\text { angegeben }\end{array}$ & Angina & $\begin{array}{l}\text { Inj.v. } 1500 \text { E.D.A. } \\
\text { Bepins. mit } \\
\text { L g golscher Lös. }\end{array}$ & 16 \\
\hline
\end{tabular}


C. Nishono:

(Fortsetzung.)

\begin{tabular}{|c|c|c|c|c|c|c|}
\hline Nr. & $\begin{array}{l}\text { Name des } \\
\text { Untersuchten } 0 . \\
\text { seine Familien- } \\
\text { beziehung zum } \\
\text { Kranken }\end{array}$ & 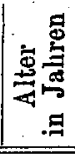 & $\begin{array}{l}\text { Wichtige } \\
\text { Anamnese }\end{array}$ & $\begin{array}{l}\text { Lokaler } \\
\text { Befund des } \\
\text { Rachens }\end{array}$ & Behandlung & $\begin{array}{c}\text { Dauer der } \\
\text { Anwesen- } \\
\text { heit des } \\
\text { Bacillus } \\
\text { in Tagen } \\
\end{array}$ \\
\hline 8 & $\begin{array}{l}\text { Tetsu, } \\
\text { GroBmutter }\end{array}$ & 48 & $\begin{array}{l}\text { litt niemals } \\
\text { an Rachen- } \\
\text { krankheit }\end{array}$ & normal & $\begin{array}{l}\text { Inj. v. } \\
1500 \text { E. D. A. } \\
\text { Bepins. mit } \\
\text { Arg. nitr. }\end{array}$ & $\begin{array}{c}\text { nicht } \\
\text { weiter } \\
\text { untersucht }\end{array}$ \\
\hline 9 & $\begin{array}{l}\text { Fumi, } \\
\text { Schwester }\end{array}$ & $\mathbf{5}$ & $\begin{array}{l}16 \text { Tage vor- } \\
\text { her erkrankt } \\
\text { an Diphtherie }\end{array}$ & , & $\begin{array}{c}\text { nicht } \\
\text { behandelt }\end{array}$ & 6 \\
\hline 10 & 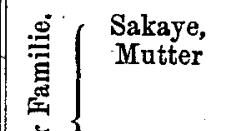 & 36 & $\begin{array}{l}\text { litt im Kindes- } \\
\text { alter an Diph- } \\
\text { therie }\end{array}$ & . & $\begin{array}{l}\text { Inj. v. } \\
1500 \text { E. D. A. } \\
\text { Bepins. mit } \\
\text { Arg. nitr. }\end{array}$ & 7 \\
\hline 11 & $\begin{array}{c}\text { Kiku, } \\
. \\
\text { Dienerin }\end{array}$ & 20 & $\underset{\substack{\text { litt nie an } \\
\text { Rachenkrank- } \\
\text { heit }}}{ }$ & " & desgl. & 9 \\
\hline 12 & $\begin{array}{l}\text { Shin, } \\
\text { Matter }\end{array}$ & 41 & $\begin{array}{l}\text { habituelle } \\
\text { Heiserkeit }\end{array}$ & $\underset{\text { injiziert }}{\text { nur leicht }}$ & $\begin{array}{l}\text { nicht } \\
\text { behandelt }\end{array}$ & $\begin{array}{c}\text { nicht } \\
\text { weiter } \\
\text { untersucht }\end{array}$ \\
\hline 13 & $\begin{array}{l}\text { Hisa, } \\
\text { Mutter }\end{array}$ & 37 & $\begin{array}{c}\text { litt nie an } \\
\text { Rashenkrank- } \\
\text { heit }\end{array}$ & desgl. & $\begin{array}{l}\text { Inj. } \mathbf{~} \\
\text { 1500 E. D. A. } \\
\text { Bepins. mit } \\
\text { Loefflers M. }\end{array}$ & 25 \\
\hline 14 & $\begin{array}{c}\text { Sada, } \\
\text { Schwester }\end{array}$ & 6 & $\begin{array}{l}\text { im 2. Lebens- } \\
\text { jahre Keuch- } \\
\text { husten }\end{array}$ & $\begin{array}{c}\text { Angina } \\
\text { (am nächsten } \\
\text { Tage Pseudo- } \\
\text { membran) }\end{array}$ & desgl. & 10 \\
\hline 15 & 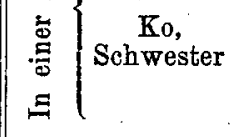 & 14 & $\begin{array}{c}\text { litt nie an } \\
\text { Rachenkrank- } \\
\text { heit }\end{array}$ & normal & $\begin{array}{l}\text { Inj. v. } \\
1500 \text { E. D. A. } \\
\text { Bepins. mit } \\
\text { Arg. nitr. }\end{array}$ & 3 \\
\hline 16 & Schester & 11 & desgl. & " & $\begin{array}{l}\text { Inj. v. } \\
1500 \text { E. D. A. } \\
\text { Bepins. mit } \\
\text { Loefflers M. }\end{array}$ & 7 \\
\hline 17 & $=\begin{array}{c}\text { Soji, } \\
\text { Bruder }\end{array}$ & 17 & ", & ", & desgl. & $\begin{array}{c}\text { über } \\
7\end{array}$ \\
\hline 18 & $\underset{\text { Mutter }}{\text { Kin, }}$ & 27 & " & nur injiziert & $\begin{array}{c}\text { nicht } \\
\text { behandelt }\end{array}$ & 12 \\
\hline 19 & $\begin{array}{l}\text { Teizo, } \\
\text { Diener }\end{array}$ & 15 & " & desgl. & $\begin{array}{c}\text { Inj.v. } 1500 \text { E.D.A. } \\
\text { Bepins. mit } \\
\text { Arg. nitr. }\end{array}$ & 17 \\
\hline 20 & $\begin{array}{l}\text { Mitsu, } \\
\text { Schwester der } \\
\text { GroBmutter }\end{array}$ & 62 & $\begin{array}{c}\text { seit einigen } \\
\text { Jahren habi- } \\
\text { tuelle Heiserk. }\end{array}$ & " & desgl. & 4 \\
\hline 21 & $\begin{array}{l}\text { Natsu, } \\
\text { Mutter }\end{array}$ & 24 & $\begin{array}{c}\text { nicht } \\
\text { angegeben }\end{array}$ & normal & $\begin{array}{c}\text { nicht } \\
\text { behandelt }\end{array}$ & $\begin{array}{c}\text { nicht } \\
\text { weiter } \\
\text { untersucht }\end{array}$ \\
\hline
\end{tabular}


Untersuchung der Hausgenossen von Diphtheriekranken.

(Fortsetzung.)

\begin{tabular}{|c|c|c|c|c|c|c|}
\hline Nr. & $\begin{array}{c}\text { Name des } \\
\text { Untersuchten u. } \\
\text { seine Familien- } \\
\text { beziehung zum } \\
\text { Kranken }\end{array}$ & 总总 & $\begin{array}{l}\text { Wichtige } \\
\text { Anamnese }\end{array}$ & $\begin{array}{l}\text { Lokaler } \\
\text { Befund des } \\
\text { Rachens }\end{array}$ & Behandlung & $\begin{array}{l}\text { Dauer der } \\
\text { Anwesen- } \\
\text { heit des } \\
\text { Bacillus } \\
\text { in Tagen }\end{array}$ \\
\hline 22 & $\begin{array}{c}\text { Hana, } \\
\text { Dienerin }\end{array}$ & 28 & $\begin{array}{l}\text { nicht } \\
\text { angegeben }\end{array}$ & $\begin{array}{c}\text { nur } \\
\text { angeschw. }\end{array}$ & $\begin{array}{c}\text { nicht } \\
\text { behandelt }\end{array}$ & $\begin{array}{c}\text { nicht } \\
\text { weiter } \\
\text { untersucht }\end{array}$ \\
\hline 23 & $\begin{array}{c}\text { Kiyo, } \\
\text { Schwester }\end{array}$ & 9 & $\begin{array}{c}\text { litt nie an } \\
\text { Rachenkrank. } \\
\text { heit }\end{array}$ & desgl. & $\begin{array}{c}\text { Inj. } \\
1500 \text { E. D. A. }\end{array}$ & 11 \\
\hline 24 & 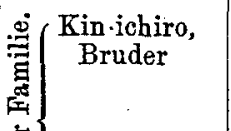 & 7 & $\begin{array}{l}\text { vor } 5 \text { Monaten } \\
\text { Keachhusten }\end{array}$ & $\begin{array}{c}\text { Mandeln stark } \\
\text { angesch w. }\end{array}$ & $\begin{array}{l}\text { Inj. v. } \\
1500 \text { E. D. A. } \\
\text { Bepins. mit } \\
\text { Arg. nitr. }\end{array}$ & $\begin{array}{l}\text { über } \\
15\end{array}$ \\
\hline 25 & $\begin{array}{l}\text { Senjiro } \\
\text { Bruder }\end{array}$ & 4 & desgl. & $\begin{array}{l}\text { Mandeln } \\
\text { leicht } \\
\text { angeschw. }\end{array}$ & desgl. & 11 \\
\hline 26 & $\begin{array}{l}\text { Chiyono, } \\
\text { Dienerin }\end{array}$ & 18 & $\begin{array}{c}\text { niemals } \\
\text { Rachenkrank- } \\
\text { heit }\end{array}$ & normal & " & 5 \\
\hline 27 & $\begin{array}{l}\text { Chiyo, } \\
\text { Schwester }\end{array}$ & 13 & unklar & $\begin{array}{c}\text { keine be- } \\
\text { deatende } \\
\text { Veränderung }\end{array}$ & $\begin{array}{c}\text { nicht } \\
\text { behandelt }\end{array}$ & $\begin{array}{c}\text { nicht } \\
\text { weiter } \\
\text { untersucht }\end{array}$ \\
\hline 28 & $\begin{array}{l}\text { Chise, } \\
\text { Mutter }\end{array}$ & 41 & $\begin{array}{c}\text { litt nie an } \\
\text { Rachenkrank- } \\
\text { beit }\end{array}$ & $\begin{array}{l}\text { Mandeln } \\
\text { nur leicht } \\
\text { angeschw. }\end{array}$ & $\begin{array}{l}\text { Inj. v. } \\
1000 \text { E. D. A. } \\
\text { Bepins. mit } \\
\text { Arg. nitr. }\end{array}$ & $\begin{array}{c}\text { über } \\
10\end{array}$ \\
\hline 29 & $\begin{array}{l}\text { Suzuki, } \\
\text { Dienerin }\end{array}$ & 13 & desgl. & desgl. & $\begin{array}{c}\text { nicht } \\
\text { behandelt }\end{array}$ & $\begin{array}{l}\text { nicht } \\
\text { weiter } \\
\text { untersucht }\end{array}$ \\
\hline 30 & $\begin{array}{l}\text { Toshiwo, } \\
\text { Bruder }\end{array}$ & 7 & $\begin{array}{l}\text { hatte } 2 \text { Woch. } \\
\text { vorher unbe- } \\
\text { kannteRachen- } \\
\text { krankheit }\end{array}$ & " & $\begin{array}{l}\text { Inj. v. } \\
1000 \text { E. D. A. } \\
\text { Bepins. mit } \\
\text { Arg. nitr. }\end{array}$ & 15 \\
\hline 31 & $\begin{array}{c}\text { Kametaro, } \\
\text { Bruder }\end{array}$ & 13 & $\begin{array}{c}\text { litt nie an } \\
\text { Rachenkrank- } \\
\text { heit }\end{array}$ & $\begin{array}{c}\text { Angina } \\
\text { (nach 2 Tagen } \\
\text { Pseudo- } \\
\text { membran) }\end{array}$ & $\begin{array}{l}\text { Inj. v. } \\
2000 \text { E. D. A. } \\
\text { Bepins. mit } \\
\text { Arg. nitr. }\end{array}$ & $?$ \\
\hline 32 & $\begin{array}{l}\text { Yasu, } \\
\text { Mutter }\end{array}$ & 27 & desgl. & $\begin{array}{l}\text { nar leicht } \\
\text { angeschw. }\end{array}$ & $\begin{array}{c}\text { nicht } \\
\text { behandelt }\end{array}$ & 10 \\
\hline 33 & $\begin{array}{c}\text { Toshiwo, } \\
\text { Brader }\end{array}$ & 7 & $\begin{array}{c}\text { litt vor } \\
\text { mehreren } \\
\text { Jahren an } \\
\text { Diphtberie }\end{array}$ & $\begin{array}{l}\text { nur leicht } \\
\text { injiziert }\end{array}$ & $\begin{array}{l}\text { Inj. v. } \\
\text { 1500 E. D. A. } \\
\text { Bepins. mit } \\
\text { Arg. nitr. }\end{array}$ & 10 \\
\hline 34 & $\begin{array}{l}\text { Toyo, } \\
\text { Mutter }\end{array}$ & 38 & $\begin{array}{c}\text { litt nie an } \\
\text { Rachenkrank- } \\
\text { heit }\end{array}$ & desgl. & desgl. & 11 \\
\hline 35 & $\begin{array}{l}\text { Tadawo, } \\
\text { Bruder }\end{array}$ & 12 & desgl. & , & , & 6 \\
\hline
\end{tabular}


C. Nishuno:

(Fortsetzung.)

\begin{tabular}{|c|c|c|c|c|c|c|}
\hline $\mathrm{Nr}$. & $\begin{array}{l}\text { Name des } \\
\text { Untersuchten a. } \\
\text { seine Familien- } \\
\text { beziehung zum } \\
\text { Kranken }\end{array}$ & 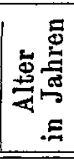 & $\begin{array}{l}\text { Wichtige } \\
\text { Anamnese }\end{array}$ & $\begin{array}{l}\text { Lokaler } \\
\text { Befund des } \\
\text { Rachens }\end{array}$ & Behandlung & $\begin{array}{l}\text { Dauer der } \\
\text { Anwesen- } \\
\text { heit des } \\
\text { Bacillus } \\
\text { in Tagen } \\
\end{array}$ \\
\hline 36 & $\begin{array}{c}\text { Haru, } \\
\text { Schwester }\end{array}$ & 9 & unklar & $\begin{array}{c}\text { keine } \\
\text { Veränderung }\end{array}$ & $\begin{array}{c}\text { nicht } \\
\text { behandelt }\end{array}$ & $\begin{array}{c}\text { nicht } \\
\text { weiter } \\
\text { nntersucht }\end{array}$ \\
\hline 37 & $\begin{array}{l}\text { Kume, } \\
\text { Mutter }\end{array}$ & 30 & $\begin{array}{c}\text { litt nie an } \\
\text { Rachenkrank- } \\
\text { heit }\end{array}$ & Angina & desgl. & 10 \\
\hline 38. & $\begin{array}{c}\text { Fumi, } \\
\text { Dienerin }\end{array}$ & 13 & . desgl. & $\underset{\text { injiziert }}{\text { nur leicht }}$ & " & 10 \\
\hline 39 & $\begin{array}{l}\text { Hasime, } \\
\text { Bruder }\end{array}$ & 8 & " & $\begin{array}{l}\text { nur stark } \\
\text { angeschw. }\end{array}$ & $\begin{array}{l}\text { Inj. v. } \\
\text { 1500 E. D. A. } \\
\text { Bepins. mit } \\
\text { Arg. nitr. }\end{array}$ & 21 \\
\hline 40 & $\begin{array}{l}\text { Fumi, } \\
\text { Matter }\end{array}$ & 29 & " & $\begin{array}{l}\text { nur leicht } \\
\text { injiziert }\end{array}$ & desgl. & 14 \\
\hline 41 & $\begin{array}{c}\text { Dai, } \\
\text { Mutter }\end{array}$ & 38 & $"$ & desgl. & $\begin{array}{c}\text { nicht } \\
\text { behandelt }\end{array}$ & $\begin{array}{c}\text { nicht } \\
\text { weiter } \\
\text { untersucht }\end{array}$ \\
\hline
\end{tabular}

Aus der Tabelle sieht man erstens, dab bei manchen Familien gleichzeitig zwei Bazillenträger vorkamen; ich erinnere hier an die Mitteilung von Scheller (12), der durch wiederholte bakteriologisehe Untersuchungen einiger Familien fast bei jedem Mitgliede Diphtheriebazillen fand und nachweisen konnte, daß beinahe sämtliche Mitglieder einer Familie, in welcher ein Diphtheriefall konstatiert werden konnte, früher oder später Diphtheriebazillen aufwiesen. So entstammen meine 41 Fälle 35 Familien, indem bei 6 Familien gleichzeitig zwei Träger sich nachweisen lieBen; der Prozentsatz der Bazillenträger enthaltenden Familien betrug also 27.6 (35:127 $=27 \cdot 6$ Prozent).

Zweitens sieht man aus der. Tabelle, daB die größere Zahl der Diphtheriebazillenträger weibliche Individuen sind. Kober fand dasselbe. Das Uberwiegen der Trägerinnen über die Träger tritt nicht nur in ihrer absoIuten Zahl, sondern auch beim Vergleich des Prozentsatzes der Trägerinnen mit dem der Träger hervor:

unter 321 männlichen Personen befanden sich 10, also 3.1 Prozent, "344 weiblichen " . " " $31,, 9.0 \quad$,

Bei Kober waren die meisten der Träger im kindlichen Alter, während bei mir, wie man drittens aus der Tabelle sieht, die Anzahl der Kinder fast gleich der der Erwachsenen ist. Wenn ich aber den Prozentsatz der unter 15 Jahren alten Träger mit dem der über 16 Jahre alten vergleiche, so überwiegen auch in meinen Fällen die ersteren: 
Unter 242 unter 15 Jahre alten Personen befanden sich 21, also 8.6 Proz., , 423 über $16 \cdot, ", ", \quad, \quad 20,, 4.7$,

Viertens sieht man, daß die Träger am häufigsten unter solchen Personen vorkommen, welche wie junge Geschwister und Mütter, sich mit Kindern riel beschäftigen. So sind unter 41 Trägern 19 Geschwister, 12 Mlütter, 7 Dienerinnen (die meisten davon gleichzeitjg Kinderwärterinnen) und 3 sonstige Familienglieder.

Fünftens ergibt sich, daß die Dauer der Anwesenheit der Diphtheriebazillen bei Trägern ziemlich verschieden ist; sie schwankt bei den meisten der wiederholt untersuchten Fälle zwischen 2 bis 25 Tagen und beträgt durehschnittlich 10 Tage. Lokale Behandlung mit Silbernitratlösung oder Loefflerschem Lokalmittel (13), sowie subkutane Injektion des Diphtherieantitoxins scheinen im ganzen keinen EinfluB auf die Dauer der Anwesenheit der Bazillen auszuäben; so sieht man z. B. den behandelten 13. Fall mit 25 tägiger Dauer und in auffallendem Gegensatz dazu den nicht behandelten 9. mit 6 tägiger Dauer.

\section{Epidemiologische Bedeutung der Diphtheriebazillenträger.}

Wenn die Infektion der Diphtherie, wie ich in der Einleitung gesagt habe, fast ausschlieBlich direzt von Mensch zu Mensch geschieht, so kommen bei der Epidemiologie der Diphtherie hauptsä,chlich der Diphtheriekranke und der Diphtheriebazillenträger in Betracht. Da nun der erstere wegen seines Krankseins naturgemäB an sein Bett gefesselt bleibt, ist das Berülrungsgebiet bzw. die Infektionsgefahr nur auf sein Zimmer oder Haus beschränkt, während bei letzterem, weil er wegen seines Gesundseins frei herumgeht, soweit nicht gesetzliche Bestimmungen dies verhindern, das Berührungsgebiet sehr viel gröBer sein muB. Daraus kann man schlieBen, wenigstens hinsichtlich der Ausdehnung des Infektionsgebietes, daB die epidemiologische Bedeutung des Diphtheriebazillenträgers gröBer als die des Diphtheriekranken selbst ist. Sie ist besonders grob, wenn die Träger Kinder oder Mütter sind, die am häufigsten mit Kindern in Berührung kommen. Diese gefährlichsten Träger fanden sich bei etwa ein Viertel der mehr als 100 untersuchten Familien. In Wirklichkeit würden sich noch mehr Träger gefunden haben, wenn ich nicht nur den Rachen untersucht hätte, weil bei manchen Leuten die Diphtheriebazillen nicht im Rachen, sondern nur in den Nasenhöhlen sich nachweisen lassen. So konnte Scheller (12) bei einem Mädchen in den Abstrichen von beiden Tonsillen niemals Diphtheriebazillen nachweisen, hingegen war es ihm möglich, sie aus dem Sekret beider Nasenhöhlen jedesmal zu züchten. 
Der Nachweis von infektionsfähigen Diphtheriebazillen an und in leblosen Gegenständen gelang zwar manchen Autoren, z. B. Abel (14) an Spielzeug, Wright und Emerson (15) in Kehricht, Park (16) an Bettbezügen und Ritter (17) an Wänden usw., aber die epidemiologische Bedeutung der Bakterien ist unter solchen Umständen wahrscheinlich viel geringfügiger, weil sie in diesen Fällen nur kürzere Lebensfähigkeit und geringere Infektionsmöglichkeit als beim Träger haben.

Nun komme ich zur Frage, wie und in welchem Grade die Hausdesinfektion bei Diphtherie einer Weiterverbreitung der Krankheit vorbeugen kann. Man wird nicht $\mathrm{zu}$ weit gehen, wenn man behauptet, daB die Hausdesinfektion bei dem gegenwärtigen Stande der Diphtherieprophy. laxis ziemlich zwecklos sei, sofern gefährliche Bazillenträger dabei unberührt bleiben.

Zum Schluß möchte ich über das Wesen der Diphtheriebazillenträger noch einige Worte hinzufügen. Ein kleiner Teil von ihnen befindet sich $\mathrm{ja}$ in der Inkubationszeit der Diphtherie; diese sind also keine Träger im eigentlichen Sinne, während die meisten von vornherein immun gegen Diphtherie sind. So hatte schon Wassermann (18) konstatiert, daB eine große Anzahl von Individuen bereits im Kindesalter mit Schutzeinrichtungen gegen die Diphtherie versehen und auf diese Weise vielleicht zeitlebens gegen Diphtherie immun ist. E. Neisser (3) hat auch bei einer Diphtheriebazillenträgerin konstatiert, daB sie in $1.0 \mathrm{com}$ Blutserum $3 / 4$ Immunisierungseinheiten Diphtherieantitoxin beherbergte. Der Diphtheriebazillenträger wird daher in den meisten Fällen eine immune Person sein, die Diphtheriebazillen bei sich trägt, die zwar nicht für sie selbst, wohl aber für andere infektionsfähig sind.

\section{Kurze Zusammenfassung.}

A. 1. Bei 35 unter 127 Familien von Diphtheriekranken, also in etwa 28 Prozent sämtlicher Familien habe ich einen oder gelegentlich gleichzeitig zwei Diphtheriebazillenträger nachgewiesen.

2. Bei 41 unter 665 Individuen dieser 127 Familien, also in etwa 6 Prozent sämtlicher Familienglieder von Diphtheriekranken habe ich echte Diphtheriebazillen im Schlunde nachgewiesen.

3. Der Prozentsatz der weiblichen Träger ist erheblich größer als der der männlichen (9:3 Prozent).

4. Der Prozentsatz der Träger unter den bis 15 Jahre alten Kindern ist fast doppelt so grob wie der unter mehr als 15 Jahre alten Personen (8.6:4.7 Prozent). 
5. In bezug auf das Familienverhältnis des Trägers kommen die Geschwister (19) am häufigsten vor, dann Mütter (12) und.endlich Dienstmädchen (7).

6. Die Dauer der Anwesenheit der Diphtheriebazillen bei Trägern beträgt durchschnittlich 10 Tage; subkutane Antitoxininjektion und gewöhnliche lokale Behandlung scheinen keinerlei EinfluB darauf auszuüben.

B. Epidemiologisch spielt der Diphtheriebazillenträger unter Umständen eine weit gröBere Rolle als der Diphtheriekranke selbst, von leblosen verunreinigten Gegenständen gar nicht zu sprechen; die epidemiologische Bedeutung solcher Träger erscheint um so gröber, als sie, wie erwiesen, besonders häufig unter Kindern und unter Frauen vorkommen, welche sich mit Kindern viel beschäftigen.

Man geht nicht zu weit, wenn man behauptet, daB, sofern solche gefährliche und weit verbreitete Diphtheriebazillenträger nicht gleich dem Kranken streng isoliert werden, der Desinfektion lebloser Gegenstände eine große prophylaktische Bedeutung nicht zuzuschreiben ist.

\section{Literatur.}

1. Flügge, Diese Zeitschrift. Bd. XVI.

2. Scheller, Handbuch der pathogenen Mikroorganismen v. Kolle-Wasserman n. Ergänz.-Bd. II. H. 2.

3. Neisser, Deutsche med. Wochenschrift. 1902. Nr. 40.

4. Fibiger, Berliner klin. Wochenschrift. 1897. Nr.35/38.

5. A a ser, Deutsche med. Wochenschrift. 1895. Nr. 22.

6. Cuno, Ebenda. 1902. Nr. 43.

7. Fisher, Journ. of American Med. Association. Vol. LII. Nr. 6.

8. Loeffler, Deutsche med. Wochenschrift. 1894. Nr. 47.

9. Müll er, Jahresbericht für Rinderheilkunde. Bd. XLIII.

10. Ustvedt, Diese Zeitschrift. Bd. LIV.

11. Kober, Ebenda. Bd. XXXI.

12. Scheller, Centralblatt für Bakteriologie. 1906. Bd. XI.

13. Loeffler, Ebenda. 1894. Bd. XVI.

14. Abel, Ebenda. 1893. Bd. XIV.

15. Wright u. Emerson, Ebenda. 1894. Bd. XVI.

16. Park, New York Med. Record. 1892.

17. Ritter, Centralblatt für Bakteriologie. 1894. Bd. XVI. (Ref.).

18. Wassermann, Diese Zeitschrift. 1895. Bd. XIX, 